\title{
ALMOST PERIODIC SOLUTIONS FOR ABEL EQUATIONS
}

\author{
ZENG WEIYAO \\ Department of Mathematics \\ Changsha Railway University \\ Changsha Hunan 410075 \\ P.R CHINA
}

\author{
SHI JINLIN \\ LIN ZHENSHENG \\ Department of Mathematics \\ Fuzhou University \\ Fuzhou Fujian 350002 \\ P R CHINA
}

and

\section{LOKENATH DEBNATH}

Department of Mathematics

University of Central Florida

Orlando, FL 32816, U.S A

(Received January 11, 1994 and in revised form April 15,1997)

\begin{abstract}
Using the Liapunov function method, the existence of almost periodic solutions of a scalar differential equation is discussed The results for the scalar differential equation are then applied to prove the existence and stability of almost periodic solutions of Abel differential equations We obtain several interesting results which improve the results due to Chongyou [1] and Dongpin [2]
\end{abstract}

KEY WORDS AND PHRASES: Abel differential equations, almost periodic solutions 1991 AMS SUBJECT CLASSIFICATION CODES: $34 C 27$

\section{INTRODUCTION}

Many results have been proved for the existence of periodic solutions for the Abel differential equation

$$
x^{\prime}(t)=a(t) x^{3}+b(t) x^{2}+c(t) x+d(t) .
$$

However, attention is hardly given to prove results on the existence of almost periodic solutions of $\left(\begin{array}{ll}1 & 1\end{array}\right)$ The main purpose of this paper is to investigate the existence and stability of almost periodic solutions of the Abel equation (1.1). First, we introduce the concept of characteristic function of equation (1 1$)$ and point out that there are relations between the characteristic function and the existence of almost periodic solutions of (1.1) In section 2, we use the Liapunov function method to prove a general theorem on almost periodic solutions of a scalar differential equation. This theorem extends the results of Fink [3], and can be used to simplify the proof of some results of Fink [3]. In section 3, the above general theorem is applied to investigate the existence and stability of almost periodic solutions of the Abel differential equations. We also obtain some interesting results which improve the results of Chongyou [1] and Dongpin [2]

\section{ALMOST PERIODIC SOLUTIONS FOR SCALAR EQUATIONS}

We consider a general scalar differential equation

$$
x^{\prime}(t)=f(t, x)
$$

where $f: R \times R \rightarrow R$, is a uniformly almost periodic function in $t$ with respect to $x \in R$ For any Liapunov function $V(t, x)$ defined on $R \times R$, we define 


$$
V_{(2.1)}^{\prime}(t, x)=\varlimsup_{k \rightarrow 0} \frac{1}{h}\{V(t+h, x+h f(t, x))-V(t, x)\} .
$$

If $x(t)$ is a solution of equation (2 1$)$, we define

$$
\dot{V}_{(21)}(t, x(t))=\varlimsup_{h \rightarrow 0^{+}} \frac{1}{h}\{V(t+h, x(t+h))-V(t, x(t))\} .
$$

It is well known that, if $V(t, x)$ satisfies the Lipschitz condition locally, then, we have

$$
\dot{V}_{(2.1)}(t, x(t))=V_{(2.1)}^{\prime}(t, x) \text {. }
$$

For equation (2.1), we have the following general theorem which improves the results of Fink [4] We follow Fink [4] to prove the following general theorem

THEOREM 2.1. Suppose $\phi(t)$ is a bounded solution of equation (2 1$)$ on $R$, and there exist almost periodic functions $\alpha(t), \beta(t)$ such that

$$
\alpha(t) \leq \phi(t) \leq \beta(t), \quad t \in R .
$$

If there is a uniformly continuous Liapunov function $V(t, x)$ satisfying

(i) $a(|x|) \leq V(t, x) \leq b(|x|)$, where $a(r), b(r)$ are continuous and increasing functions with $a(0)=b(0)=0$,

(ii) $\dot{V}_{(21)}(t, x(t)-\phi(t)) \leq 0$, for $\alpha(t) \leq x(t) \leq \beta(t$, ) $t \in R$, then, equation (2 1) has a unique almost periodic solution $x(t)$ satisfying the inequality

$$
\alpha(t) \leq x(t) \leq \beta(t)
$$

with $\bmod (x) \subset \bmod (\alpha, \beta, f)$ and $x(t)$ is uniformly stable.

Note that Theorem 12.8 of Fink [4] can be obtained from Theorem 2.1

THEOREM 2.2. Suppose $f(t, x)$ is a decreasing function in $x$ If equation (2 1) admits bounded solutions, equation (2 1) has an almost periodic solution $x(t)$ with $\bmod (x) \subset \bmod (f)$

PROOF. Suppose $\phi(t)$ is a bounded solution of (2.1) Let $\alpha=\inf _{t \in R} \phi(t), \beta=\sup _{t \in R} \phi(t) \quad$ Taking a Liapunov function $V(t, x)=\left(1 / 2 x^{2}\right)$, we have the following

$$
\dot{V}_{(21)}(t, x(t)-\phi(t))=(x(t)-\phi(t))[f(t, x(t))-f(t, \phi(t))] \leq 0, \quad \text { for } \quad \alpha \leq x(t) \leq \beta .
$$

All conditions of Theorem 2.1 are satisfied, so equation (21) possesses an almost periodic solution $\tilde{x}(t)$ with $\bmod (\tilde{x}) \subset \bmod (f)$

LEMMA 2.1. Suppose conditions of Theorem 2.1 are satisfied The existence of a uniformly continuous Liapunov function satisfying conditions (i), (ii) of Theorem 21 are inherited by the hull of equation (2.1).

Proof of this lemma is almost the same as the one in Fink [4], so we omit it.

PROOF OF THEOREM 2.1. For sequence $\alpha=\left\{\alpha_{n}\right\}$, and almost periodic function $f(t)$, we denote the limit $\lim _{n \rightarrow \infty} f\left(t+\alpha_{n}\right)$ by $T_{\alpha} f$. Let $a=\inf _{t \in R^{-}} \phi(t), b=\sup _{t \in R^{-}} \phi(t)$ and construct functions $\tilde{\alpha}(t)=\max (\alpha(t), a), \tilde{\beta}(t)=\min (\beta(t), b)$, then

$$
\alpha(t) \leq \tilde{\alpha}(t) \leq \phi(t) \leq \tilde{\beta}(t) \leq \beta(t), \quad \text { for } \quad t \in R^{-} .
$$

Now, we shall prove that equation (2.1) has and only has a solution $\phi(t)$ such that

$$
\tilde{\alpha}(t) \leq \phi(t) \leq \tilde{\beta}(t), \quad t \in R^{-} .
$$

Suppose equation (2 1) has another solution $\phi(t)$ such that

$$
\tilde{\alpha}(t) \leq \psi(t) \leq \tilde{\beta}(t), \quad t \in R^{-} .
$$


Without loss of generality, one may assume $\psi(0)>\phi(0)$, then $\psi(t)>\phi(t)$ for $t \in R$ Since $b=\sup _{t \in R^{-}} \phi(t)$, there exists a sequence $\left\{t_{n}\right\}$ such that $t_{n} \rightarrow t^{\prime}$ as $n \rightarrow \infty\left(t^{\prime}\right.$ may be $\left.-\infty\right)$ and $\lim _{n \rightarrow \infty} \phi\left(t_{n}\right)=b$ Since

$$
\phi(t) \leq \psi(t) \leq b, \quad t \in R^{-},
$$

we have

$$
\phi\left(t_{n}\right)<\psi\left(t_{n}\right) \leq b
$$

We obtain $\lim _{n \rightarrow \infty} \psi\left(t_{n}\right)=b$ Hence

$$
\lim _{n \rightarrow \infty}\left(\psi\left(t_{n}\right)-\phi\left(t_{n}\right)\right)=0
$$

On the other hand, by (i), we have

$$
a\left(\left|\psi\left(t_{n}\right)-\phi\left(t_{n}\right)\right|\right) \leq V\left(t_{n}, \psi\left(t_{n}\right)-\phi\left(t_{n}\right)\right) \leq b\left(\left|\psi\left(t_{n}\right)-\phi\left(t_{n}\right)\right|\right),
$$

hence

$$
\lim _{n \rightarrow \infty} V\left(t_{n}, \psi\left(t_{n}\right)-\phi\left(t_{n}\right)\right)=0
$$

Since

$$
\dot{V}_{(2.1)}(t, \psi(t)-\phi(t)) \leq 0,
$$

$V(t, \psi(t)-\phi(t))$ is bounded and decreasing in $t$, which implies that the limit

$$
\lim _{t \rightarrow t^{\prime}} V(t, x(t)-\phi(t))
$$

exists It follows from above that

$$
\lim _{n \rightarrow \infty} V\left(t_{n}, x\left(t_{n}\right)-\phi\left(t_{n}\right)\right)=0,
$$

therefore,

$$
\lim _{t \rightarrow t^{\prime}} V\left(t, \psi(t)-\phi\left(t_{1}\right)\right)=0 .
$$

On the other hand, $V(t, \psi(t)-\phi(t)) \geq 0$ and is decreasing in $t$, hence

$$
V(t, \psi(t)-\phi(t)) \equiv 0, \quad t_{1} \leq t \leq 0 .
$$

and $\psi(t) \equiv \phi(t)$ for $t_{1} \leq t \leq 0$ Since equation (2.1) has a unique solution with the initial value, $\psi(t) \equiv \phi(t), t \in R$ So, we prove equation (2 1) has a unique solution $\phi(t)$ satisfying

$$
\tilde{\alpha}(t) \leq \phi(t) \leq \tilde{\beta}(t), \quad t \in R^{-} .
$$

For given sequences $\alpha=\left\{\alpha_{n}\right\}$, and $\beta=\left\{\beta_{n}\right\}$, since $\tilde{\alpha}(t), \tilde{\beta}(t), f(t, x)$ are almost periodic, without loss of generality, we may assume that

$$
\begin{aligned}
& T_{\alpha+\beta} f=T_{\alpha} T_{\beta} f=g \\
& T_{\alpha+\beta} \tilde{\alpha}=T_{\alpha} T_{\beta} \tilde{\alpha}=\tilde{a} \\
& T_{\alpha+\beta} \tilde{\beta}=T_{\alpha} T_{\beta} \tilde{\beta}=\tilde{b}
\end{aligned}
$$

uniformly in $t \in R$ By the same process as above and Lemma 21 , we can show that equation

$$
x^{\prime}(t)=g(t, x)
$$

admits a unique solution $x(t)$ satisfying

$$
\tilde{a}(t) \leq x(t) \leq \tilde{b}(t), \quad t \in R .
$$


Without loss of generality, we consider that $T_{\alpha+\beta} \phi$ and $T_{\alpha} T_{\beta} \phi$ are solutions of equation (2 2) It is easy to verify that

$$
\begin{array}{ll}
\tilde{a}(t) \leq T_{\alpha+\beta} \phi \leq \tilde{b}(t), & t \in R \\
\tilde{a}(t) \leq T_{\alpha} T_{\beta} \phi \leq \tilde{b}(t), & t \in R
\end{array}
$$

hence, $T_{\alpha+\beta} \phi=T_{\alpha} T_{\beta} \phi$, because equation (2 2) has a unique solution $x(t)$ satisfying

$$
\tilde{a}(t) \leq x(t) \leq \tilde{b}(t) .
$$

This implies that $\phi(t)$ is almost periodic with $\bmod (\phi) \subset \bmod (f, \alpha, \beta)$ by making reference to Fink [3] This completes the proof of Theorem 21

\section{ALMOST PERIODIC SOLUTIONS OF ABEL'S EQUATIONS}

For the Abel differential equation (1 1), we define $F(t, x)=3 a(t) x^{2}+2 b(t) x+c(t)$ as a characteristic function of equation (1.1).

Now, we investigate the existence of almost periodic solutions of the Abel differential equation $\left(\begin{array}{ll}1 & 1\end{array}\right)$ for the following cases

Case $1 b^{2}(t)-3 a(t) \cdot c(t) \leq 0$, and Case 2. $b^{2}(t)-3 a(t) \cdot c(t) \geq 0$

For Case 1, we have the following theorem

THEOREM 3.1. Let $a(t), b(t), c(t)$ and $d(t) \in A P(R)=\{f(t) \mid f(t)$ is almost periodic in $t \in R\} \quad$ If $\sup _{t \in \mathbb{R}}\left(b^{2}(t)-3 a(t) \cdot c(t)\right)<0, a(t) \neq 0$, then, the Abel differential equation

$$
x^{\prime}(t)=a(t) x^{3}+b(t) x^{2}+c(t) x+d(t)
$$

admits a unique uniformly asymptotically stable and almost periodic solution $x(t)$ with $\bmod (x) \subset$ $\bmod (a, b, c, d)$.

PROOF. We assume $a(t)<0, t \in R$, otherwise, let $t=-\tau$ Since

$$
\begin{gathered}
\sup _{t \in R}\left[b^{2}(t)-3 a(t) c(t)\right]<0 \\
F(t, x)=3 a(t) x^{2}+2 b(t) x+c(t) \leq-\frac{\inf _{t \in R}\left(3 a(t) c(t)-b^{2}(t)\right)}{3 \sup _{t \in R}|a(t)|}=:-m_{0}
\end{gathered}
$$

where $m_{0}>0$ We take a Liapunov function $\mathrm{V}(t, x, y)=\frac{1}{2}(x-y)^{2}$, and consider equations

$$
\begin{aligned}
& x^{\prime}(t)=a(t) x^{3}+b(t) x^{2}+c(t) x+d(t), \\
& y^{\prime}(t)=a(t) y^{3}+b(t) y^{2}+c(t) y+d(t),
\end{aligned}
$$

then

$$
\begin{aligned}
\dot{V}_{(3.1)}(t, x(t), y(t)) & =(x(t)-y(t))^{2} \cdot F(t, x(t)+\theta(x(t)-y(t))) \\
& \leq-m_{0}(x(t)-y(t))^{2} .
\end{aligned}
$$

It follows from Theorem 1 in Weiyao [6] that the Abel differential equation (1 1 ) admits a unique almost periodic solution $x(t)$ which is uniformly asymptotically stable with $\bmod (x) \subset \bmod (a, b, c, d) \quad$ This completes the proof

If condition of Theorem $3.1 \sup _{t \in R}\left(b^{2}(t)-3 a(t) \cdot c(t)\right)<0$ is weakened i e. $b^{2}(t)-3 a(t) \cdot c(t) \leq 0$, then, we have

THEOREM 3.2. Suppose $a(t), b(t), c(t)$ and $d(t) \in A P(R)$. If $b^{2}(t)-3 a(t) \cdot c(t) \leq 0$ and $\inf _{t \in R}|a(t)| \neq 0$, then the Abel differential equation (1.1) has an almost periodic solution $\phi(t)$ with $\bmod (\phi) \subset \bmod (a, b, c, d)$ 
PROOF. Since $\inf _{t \in R}|a(t)| \neq 0$, without loss of generality, we assume that $\sup _{t \in R} a(t)<0$ We can find a sufficient large constant $M>0$ such that

$$
\left.\frac{d x}{d t}\right|_{x=M}<0 \text { and }\left.\frac{d x}{d t}\right|_{x=-M}>0
$$

hence, the Abel differential equation ( 11 l) has a solution $\tilde{\phi}(t)$ satisfying

$$
-M \leq \tilde{\phi}(t) \leq M, \quad t \geq 0 .
$$

Therefore, it has a solution $\phi(t)$ satisfying

$$
-M \leq \phi(t) \leq M, \quad t \in R .
$$

Taking a Liapunov function $V(t, x)=\frac{1}{2} x^{2}$, and noting $b^{2}(t)-3 a(t) \cdot c(t) \leq 0$ and $a(t)<0$, we compute as Theorem 3.1 that

$$
\dot{V}_{(1.1)}(t, x(t)-\phi(t)) \leq 0 \text { for } \quad-M \leq x(t) \leq M
$$

It follows from Theorem 21 that the Abel differential equation (1.1) has an almost periodic solution $\phi(t)$ with $\bmod (\phi) \subset \bmod (a, b, c, d)$. This completes the proof of Theorem 3.2

Case 2. $b^{2}(t)-3 a(t) \cdot c(t) \geq 0$

We have the following results

THEOREM 3.3. Suppose $a(t), b(t), c(t)$ and $d(t) \in A P(R)$, and $b^{2}(t)-3 a(t) \cdot c(t) \geq 0$ and $\sup _{t \in \mathbb{R}} \mathrm{a}(t)<0$. If

$$
\frac{2 b^{3}-9 a b c+\left(2 b^{2}-6 a c\right) \sqrt{b^{2}-3 a c}}{27 a^{2}}+d>\frac{d}{d t}\left(-\frac{b+\sqrt{b^{2}-3 a c}}{3 a}\right)
$$

then, the Abel differential equation (1 1 ) admits almost periodic solution $\phi(t)$ with $\bmod (\phi) \subset$ $\bmod (a, b, c, d)$, and

$$
x(t) \geq \frac{-b(t)-\sqrt{b^{2}(t)-3 a(t) \cdot c(t)}}{3 a(t)} .
$$

If

(ii)

$$
\frac{2 b^{3}-9 a b c+\left(6 a c-8 b^{2}\right) \sqrt{b^{2}-3 a c}}{27 a^{2}}+d<\frac{d}{d t}\left(\frac{-b+\sqrt{b^{2}-3 a c}}{3 a}\right)
$$

then, Abel differential equation (1 1 ) has an almost periodic solution $x(t)$ with $\bmod (x) \subset \bmod (a, b, c, d)$ and $x(t) \leq \frac{-b(t)+\sqrt{b^{2}(t)-3 a(t) \cdot c(t)}}{3 a(t)}$

PROOF FOR CASE (ii). We only prove (ii) Similarly, we can show (i) Since $\sup _{t \in R} a(t)<0$, we can find a sufficiently large $M>0$ such that

$$
\left.\frac{d x}{d t}\right|_{x=M}<0
$$

On the other hand,

$$
\begin{aligned}
\left.\frac{d x}{d t}\right|_{x=\frac{-b+\sqrt{b^{2}-3 a c}}{3 a}} & =\frac{2 b^{3}-9 a b c+\left(-8 b^{2}+6 a c\right) \sqrt{b^{2}-3 a c}}{27 a^{2}}+d \\
& <\frac{d}{d t}\left(\frac{-b+\sqrt{b^{2}-3 a c}}{3 a}\right)
\end{aligned}
$$


which implies that the Abel differential equation (1 1 ) has a solution $x(t)$ satisfying

$$
-M \leq x(t) \leq \frac{-b+\sqrt{b^{2}-3 a c}}{3 a}, \quad t \geq 0 .
$$

Since $\frac{-b+\sqrt{b^{2}-3 a c}}{3 a}$ is almost periodic, the Abel differential equation (1 1 ) has a solution $\phi(t)$ such that

$$
-M \leq \phi(t) \leq \frac{-b+\sqrt{b^{2}-3 a c}}{3 a}, \quad t \in R
$$

We take a Liapunov function $V(t, x)=\frac{1}{2} x^{2}$, then, when $-M \leq x(t) \leq \frac{-b+\sqrt{b^{2}-3 a c}}{3 a}$,

$$
\dot{V}_{(21)}(t, x(t)-\phi(t))=(x(t)-\phi(t))^{2} F(t, \zeta(t)) \leq 0\left(-M \leq \zeta(t) \leq \frac{-b+\sqrt{b^{2}-3 a c}}{3 a}\right)
$$

because, when $-M \leq x(t) \leq \frac{-b+\sqrt{b^{2}-3 a c}}{3 a}$, we find

$$
F(t x,(t))=3 a(t) x^{2}+2 b(t) x(t)+c(t) \leq 0 .
$$

It follows from Theorem 21 that the Abel differential equation (1.1) has an almost periodic solution $\phi(t)$ with $\bmod (\phi) \subset \bmod (a, b, c, d)$ Similarly, we can show the second part of the theorem The proof of the theorem is complete

From Theorem 3 , we can get some new results

THEOREM 3.4. Suppose $b(t) \equiv 0, a(t), c(t)$ and $d(t) \in A P(R)$, and $\sup _{t \in R} a(t)<0, c(t)>0$ If

$$
-\frac{2 c(t) \sqrt{-3 a(t) \cdot c(t)}}{9 a(t)}+d(t)>\frac{d}{d t}\left(-\frac{\sqrt{-3 a c}}{3 a}\right)
$$

or

(ii) $\frac{2 c(t) \sqrt{-3 a(t) \cdot c(t)}}{9 a(t)}+d(t)<\frac{d}{d t}\left(-\frac{\sqrt{-3 a c}}{3 a}\right)$,

then the Abel differential equation

$$
x^{\prime}(t)=a(t) x^{3}+c(t) x+d(t)
$$

has an almost periodic solution $x(t)$ with $\bmod (x) \subset \bmod (a, c, d)$.

THEOREM 3.5. Suppose $a(t), b(t), c(t)$ and $d(t) \in A P(R)$ and $b^{2}(t)-3 a(t) \cdot c(t) \geq 0$ and $\sup _{t \in R} a(t)<0$ If

$$
\begin{aligned}
& \frac{2 b^{3}-9 a b c+\left(2 b^{2}-6 a c\right) \sqrt{b^{2}-3 a c}}{27 a^{2}}+d<\frac{d}{d t}\left(-\frac{b+\sqrt{b^{2}-3 a c}}{3 a}\right) \\
& \frac{2 b^{3}-9 a b c+\left(6 a c+8 b^{2}\right) \sqrt{b^{2}-3 a c}}{27 a^{2}}+d>\frac{d}{d t}\left(\frac{-b+\sqrt{b^{2}-3 a c}}{3 a}\right)
\end{aligned}
$$

then the Abel equation (1 1 ) has an almost periodic solution $x(t)$ with $\bmod (x) \subset \bmod (a, b, c, d)$

PROOF OF THEOREM 3.5. Since

$$
\left.\frac{d x}{d t}\right|_{x=-\frac{b+\sqrt{b^{2}-3 a c}}{3 a}}=\frac{2 b^{3}-9 a b c+\left(2 b^{2}-6 a c\right) \sqrt{b^{2}-3 a c}}{27 a^{2}}+d<\frac{d}{d t}\left(-\frac{b+\sqrt{b^{2}-3 a c}}{3 a}\right)
$$

and 


$$
\left.\frac{d x}{d t}\right|_{x=-\frac{b+\sqrt{b^{2}-3 a c}}{3 a}}=\frac{2 b^{3}-9 a b c+\left(6 a c-8 b^{2}\right) \sqrt{b^{2}-3 a c}}{27 a^{2}}+d>\frac{d}{d t}\left(-\frac{b+\sqrt{b^{2}-3 a c}}{3 a}\right),
$$

by the same argument as Theorem 3 3, Abel equation (1 1 ) has a solution $\phi(t)$ satısfying

$$
\frac{-b+\sqrt{b^{2}-3 a c}}{3 a} \leq \phi(t) \leq \frac{-b-\sqrt{b^{2}-3 a c}}{3 a}, \quad t \in R .
$$

We take a Liapunov function $V(t, x)=\frac{1}{2} x^{2}$ When

$$
\frac{-b+\sqrt{b^{2}-3 a c}}{3 a} \leq x(t) \leq \frac{-b-\sqrt{b^{2}-3 a c}}{3 a},
$$

we have

$$
\dot{V}_{(21)}(t, x-\phi(t))=(x(t)-\phi(t))^{2} F(t, \xi(t)) \geq 0
$$

where

$$
\frac{-b+\sqrt{b^{2}-3 a c}}{3 a} \leq \xi(t) \leq \frac{-b-\sqrt{b^{2}-3 a c}}{3 a} .
$$

Because $F(t, x(t)) \geq 0$ when $\frac{-b+\sqrt{b^{2}-3 a c}}{3 a} \leq x(t) \leq \frac{-b-\sqrt{b^{2}-3 a c}}{3 a}$, it follows from Theorem 31 that the Abel differential equation (1.1) has an almost periodic solution $\phi(t)$ with $\bmod (\phi) \subset \bmod (a, b, c, d)$ This proves Theorem 3.5

Now, we consider the Riccati equation

$$
x^{\prime}(t)=a(t) x^{2}+b(t) x+c(t)
$$

We have the following

THEOREM 3.6. Suppose $a, b, c \in A P(R)$ If

$$
\sup _{t \in R} a(t)<0 \quad \text { and } \quad c(t)-\frac{b^{2}(t)}{4 a(t)}>\frac{d}{d t}\left(-\frac{b(t)}{2 a(t)}\right)
$$

then, equation (3 3) has an almost periodic solution $x(t)$ with $x(t) \geq-\frac{b(t)}{2 a(t)}$.

PROOF. (i). Since $\sup _{t \in R} a(t)<0$ and $c(t)-\frac{b^{2}(t)}{4 a(t)}>0$, we can find a sufficiently large constant $M>0$ such that

$$
\left.\frac{d x}{d t}\right|_{x=M}<0
$$

and

$$
\left.\frac{d x}{d t}\right|_{x=-\frac{b(t)}{2 a(t)}}=c(t)-\frac{b^{2}(t)}{4 a(t)} \geq \frac{d}{d t}\left(-\frac{b(t)}{2 a(t)}\right)
$$

which implies that equation (3.3) has a solution $x(t)$ satisfying

$$
-\frac{b(t)}{2 a(t)} \leq x(t) \leq M, \quad t \in R
$$

We construct a Liapunov function $V(t)=\frac{1}{2} x^{2}$ When $-\frac{b(t)}{2 a(t)} \leq x(t) \leq M$, we have

$$
2 a(t) \cdot x(t)+b(t) \leq 0
$$

and 


$$
\dot{V}_{(33)}(t, x(t)-\phi(t))=2 a(t) \xi(t)+b(t) \leq 0
$$

where

$$
-\frac{b(t)}{2 a(t)} \leq \xi(t) \leq M
$$

Hence, equation (3 3) has an almost periodic solution $x(t)$ with $\bmod (x) \subset \bmod (a, b, c)$ from Theorem 21 This proves Theorem 36

We consider equation

$$
x^{\prime}(t)=-h(x)+g(t) x+f(t) .
$$

The Abel differential equation is a special case of $(34)$ We have the following results

THEOREM 3.7. Suppose $f(t), g(t) \in A P(R)$ If

(i) $h^{\prime}(x) \geq 0, \quad \lim _{x \rightarrow \pm \infty} \frac{x}{h(x)}=0$,

(ii) $\int_{0}^{t} g(s) d s$ is bounded for $t \in R$, then, equation (34) has an almost periodic solution $x(t)$ with $\bmod (x) \subset \bmod (f, g)$

PROOF. For sufficiently large $|x|$, equation (3 4) can be written in the form

$$
\frac{d x}{d t}=h(x)\left[-1+\frac{x}{h(x)} g(t)+\frac{f(t)}{h(x)}\right] .
$$

Since $\lim _{x \rightarrow \pm \infty} \frac{x}{h(x)}=0$ and $h^{\prime}(x) \geq 0$, there exists a sufficiently large $K>0$ such that

$$
\left.\frac{d x}{d t}\right|_{x=-K}>0 \text { and }\left.\frac{d x}{d t}\right|_{x=K}<0,
$$

hence, equation ( 34 ) has a solution $\phi(t)$ satisfying

$$
-K \leq \phi(t) \leq K, \quad t \in R .
$$

We construct a Liapunov function $V(t, x)=x^{2} \exp \left\{-2 \int_{0}^{t} g(s) d s\right\}$, then

$$
\begin{aligned}
\dot{V}_{(34)}(t, x(t)-\phi(t))= & 2(x(t)-\phi(t)) \exp \left(-2 \int_{0}^{t} g(s) d s\right)\{-h(x(t))+h(\phi(t))+g(t)[x(t)-\phi(t)]\} \\
& -2(x(t)-\phi(t))^{2} g(t) \exp \left(-2 \int_{0}^{t} g(s) d s\right) \\
= & -(x(t)-\phi(t))^{2} \exp \left(-2 \int_{0}^{t} g(s) d s\right) h^{\prime}(\xi(t))
\end{aligned}
$$

where $-K \leq \xi(t) \leq K$. It follows from Theorem 2.1 that equation (34) has an almost periodic solution $\phi(t)$ with $\bmod (\phi) \subset \bmod (f, g)$ The proof of Theorem 37 is complete

THEOREM 3.8. Suppose $f(t), g(t) \in A P(R)$. If

(i) $h^{\prime}(x) \geq 0$,

(ii) there is $\epsilon>0$ such that $\int_{0}^{t}(2 g(s)+\epsilon) d s$ is bounded, then, equation (3.4) admits an almost periodic solution $x(t)$ with $\bmod (x) \subset \bmod (f, g)$

PROOF. We construct a Liapunov function $V(t, x)=x^{2} \exp \left\{-\int_{0}^{t}(2 g(s)+\epsilon) d s\right\}$ Then, we have, 


$$
\begin{aligned}
V_{(34)}^{\prime}(t, x) & =-2 x \exp \left\{-\int_{0}^{t}(2 g(s)+\epsilon) d s\right\} \cdot\left[-f(t)+h(x)-h(0)+h(0)+\frac{\epsilon}{2} x\right] \\
& =-2 \exp \left\{-\int_{0}^{t}(2 g(s)+\epsilon) d s\right\} \cdot\left[h^{\prime}(\xi) x+\frac{\epsilon}{2} x+h(0)-f(t)\right] \\
& \leq \epsilon V(t, x)+2|h(0)-f(t)| \cdot \exp \left\{-\int_{0}^{t}(2 g(s)+\epsilon) d s\right\} \cdot|x| .
\end{aligned}
$$

Let $L=2 \sup _{t \in R}|h(0)-f(t)| \exp \left\{-\frac{1}{2} \int_{0}^{t}(2 g(s)+\epsilon) d s\right\}$, then

$$
\begin{aligned}
V_{(34)}^{\prime}(t, x) & \leq-\epsilon V(t, x)+L|X| \exp \left\{-\frac{1}{2} \int_{0}^{t}(2 g(s)+\epsilon) d s\right\} \\
& =-\epsilon V(t, x)+L \sqrt{V(t, x)} .
\end{aligned}
$$

Hence, if $V(0, x(0)) \leq \frac{L^{2}}{\epsilon^{2}}$, then

$$
V(t, x(t)) \leq \frac{L^{2}}{\epsilon^{2}}, \quad t \geq 0
$$

This implies that equation (34) has a bounded solution $x(t)$ on $R$ We construct a Liapunov function $W(t, x)=(x-\phi(t))^{2} \exp \left\{-\int_{0}^{t}(2 g(s)+\epsilon) d s\right\}$, by the same argument as in Theorem 36 , we obtain

$$
W_{(34)}^{\prime}(t, x) \leq-\epsilon k(x-\phi(t))^{2},
$$

where $k$ is a constant It follows from Theorem 195 in Yoshizawa [5] that equation (3 4) has an almost periodic solution $x(t)$ with $\bmod (x) \subset \bmod (f, g)$ This proves Theorem 38

\section{REFERENCES}

[1] CHONGYOU, $\mathrm{H}$, The existence of almost periodic solutions of Abel differential equations, Ann. Diff. Eqs. 1 (1985), 27-41

[2] DONGPING, J, Almost periodic solutions of equation $x^{\prime}=x^{3}+\lambda g(t) x+\mu f(t)$ and their stability, Chinese Ann. Math. 6B (1985), 185-198

[3] FINK, A M., Almost Perıodic Differentıal Equatıons, Springer-Verlag, Berlin, 1978

[4] FINK, A M, Liapunov functions and almost periodic solutions for almost periodic systems, J. Diff. Eqs. 5 (1969), 307-313

[5] YOSHIZAWA, T, Stablity Theory and the Existence of Periodic Solutions and Almost Periodic Solutions, Springer-Verlag, Berlin, 1975

[6] WEIYAO, Z., The existence of almost periodic solution via Liapunov, functions, Ann. Diff. Eqs. 7 (1991), 476-481 


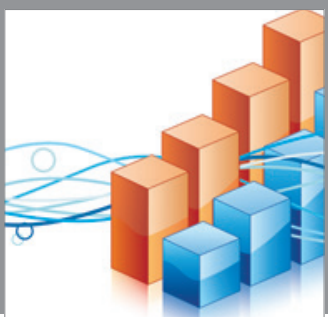

Advances in

Operations Research

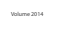

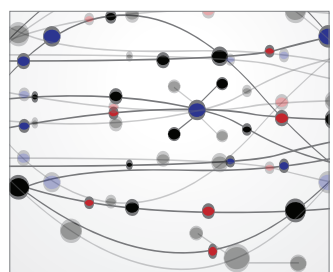

\section{The Scientific} World Journal
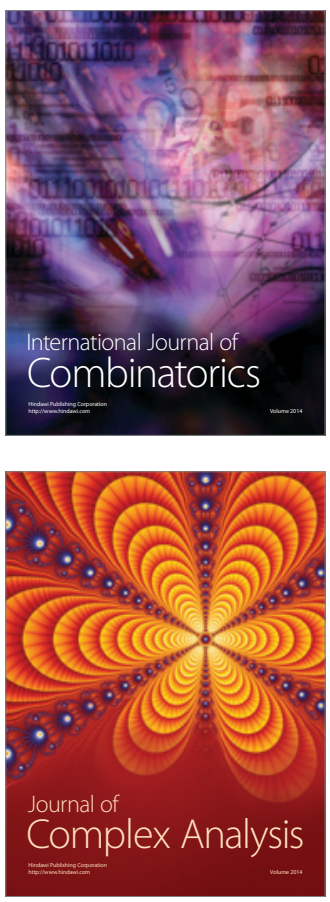

International Journal of

Mathematics and

Mathematical

Sciences


Journal of

Applied Mathematics
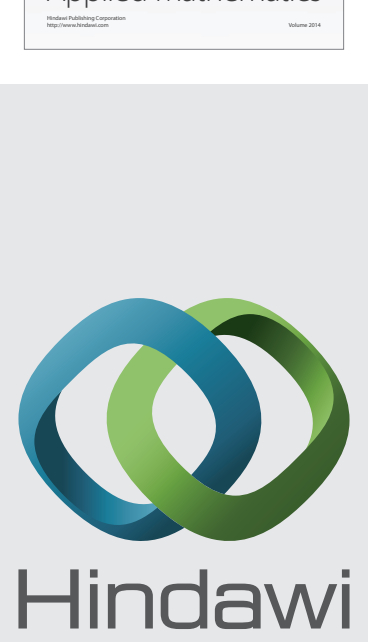

Submit your manuscripts at http://www.hindawi.com
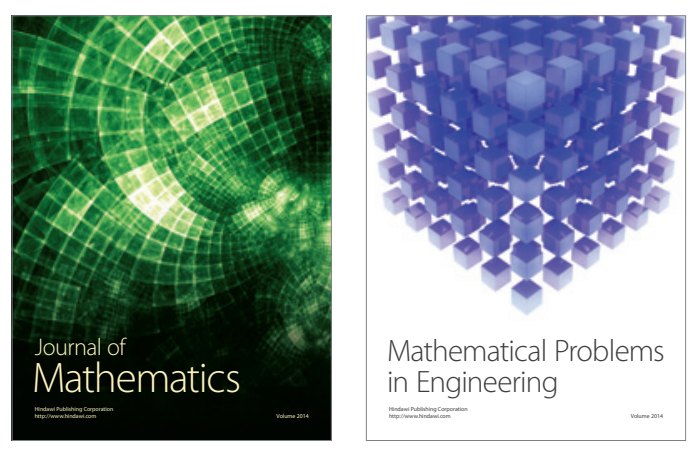

Mathematical Problems in Engineering
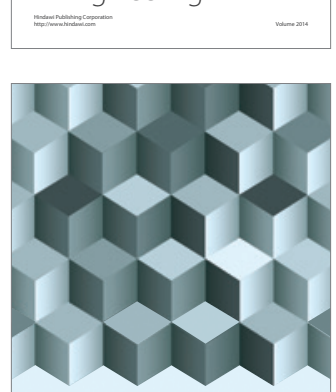

Journal of

Function Spaces
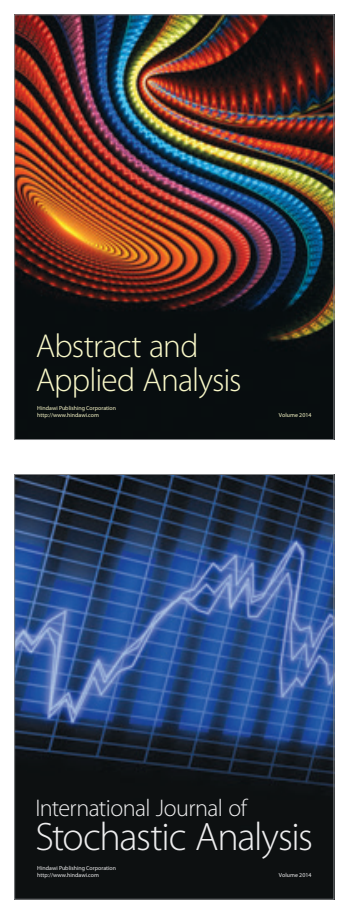

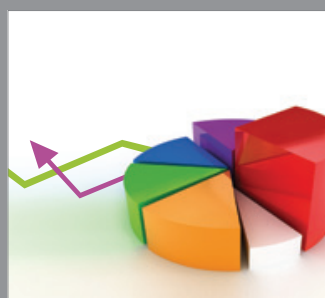

ournal of

Probability and Statistics

Promensencen
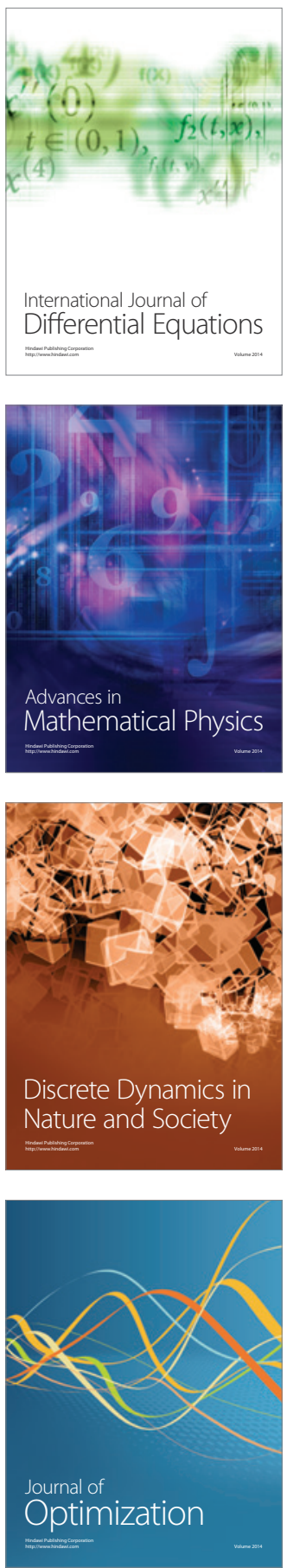